\title{
RESEARCH OF THE EFFICIENCY OF THE WIRELESS POWER TRANSFER WITH THE EMPLOYMENT OF DD INDUCTANCE COILS
}

\author{
Alexander Krainyukov ${ }^{1}$, Igor Lyaksa ${ }^{2}$, Rodions Saltanovs ${ }^{3}$ \\ 1,2Transport and Telecommunications Institute \\ Riga , Latvia, Lomonosova iela 1, LV-1019 \\ ${ }^{1}$ Ph.:+371 67100634, e-mail: Krainukovs.A@tsi.lv \\ ${ }^{2}$ Ph.:+37167100634, e-mail: ljaksa.i@tsi.lv \\ ${ }^{3} R S$ Factor \\ Ogre, Latvia \\ Ph.:+371 27178331, e-mail: r.saltanovs@transfoelectric.com
}

\begin{abstract}
The paper is devoted to using of DD inductance coils for the wireless power transfer. The aim of the given research is to determine influence of the parameters of resonance transformer on the efficiency of the wireless power transfer with the use of the DD inductance coils. Experimental installation of the wireless power transfer by a resonance inductive method was constructed. Experiments were performed with it help. Research results show influence of the distance between the coils of inductance, of the resonance transformer frequency, of the storage source voltage and of the temperature conditions on the efficiency of the wireless power transfer.
\end{abstract}

Keywords: DD inductance coil, wireless power transfer, resonance transformers, resonance inductive power transfer

\section{Introduction}

Currently, the technologies wireless power transmission for charging the automobile accumulators are given sufficient attention (Wu and other, 2011; Miller and other, 2012; Covic and other, 2013; Li and other, 2015). Charging the accumulating batteries on the move seems very perspective which is confirmed by the standards of the leading world countries (Pereirinha, 2013). But the technology of wireless power transmission for moving transport means is yet at the stage of the prototypes research and development. To charge the accumulating batteries with the wireless electro power transmission there is employed the method of electromagnetic induction.

To increase the efficiency of the systems of wireless power transmission with the electromagnetic inductance and the distance between the transmitting and receiving windings of the transformer, there is employed the technology of the resonance inductive power transfer (Kurs and other, 2007; Grajski and other, 2012). Its essence lies in using two circuits adjusted at one resonance frequency and forming a single resonance transformer. To excitate the oscillating contour of the transmitting winding of the transformer there are used the non-sinusoidal impulses. For developing resonance transformers there are used flat oneside coils of inductance: inductance coils - DD inductance coils and ring inductance coils (Budhia and other, 2013).

The main characteristic of the efficiency of the wireless system of power transmission is the efficiency which characterizes the part of power coming from the power source to the system load (power storage). The values of the wireless power transmission efficiency must be close to 1 ( $\mathrm{Li}$ and other, 2015). From the analysis of the principle of work of the wireless power transmission system, based on the resonance inter-induction of the transformer circuits, we can conclude that the efficiency of such system will be greatly dependent on the following factors:

- construction of the inductance coils of the resonance circuits of the transformer;

- distance between the inductance coils of the receiving and the transmitting circuits of the resonance transformer;

- methods of their excitation.

The advantage of using the DD coils of inductance is determined by their construction, size, and the configuration of the magnetic field which they form. There are some publications dedicated to the modelling of the wireless power transmission with the use of the DD inductance coils and to the research 
of the characteristics of the resonance transformers with the DD inductance coils ( $\mathrm{Li}$ and other, 2015; Covic and other, 2013). Though, the existing evaluations of the efficiency of employing the DD coils of inductance have been received without the account of the following factors:

- Value of clearance of the existing auto transport means;

- Influence of change of distance between the DD inductance coils on the efficiency of the wireless power transfer;

- Influence of the capacity source on the efficiency of wireless power transmission;

- Temperature conditions of work of the wireless power transmission system.

The aim of the given research is to determine influence of the distance between the inductance coils, of the resonance transformer frequency, of the storage source voltage and of the temperature conditions on the efficiency of the wireless power transmission with the use of the DD inductance coils.

\section{Experimental installation of the wireless power transfer by a resonance inductive method}

To conduct research of the peculiarities of the resonance induction method of wireless transmission there has been developed a structural scheme of an experimental installation of wireless power transmission. The structural scheme of the experimental installation is given in fig. 1. To develop the structural scheme of the experimental installation of wireless power transmission there has been chosen the impulse way of stimulating oscillations in the transmitting circuit of the resonance transformer.

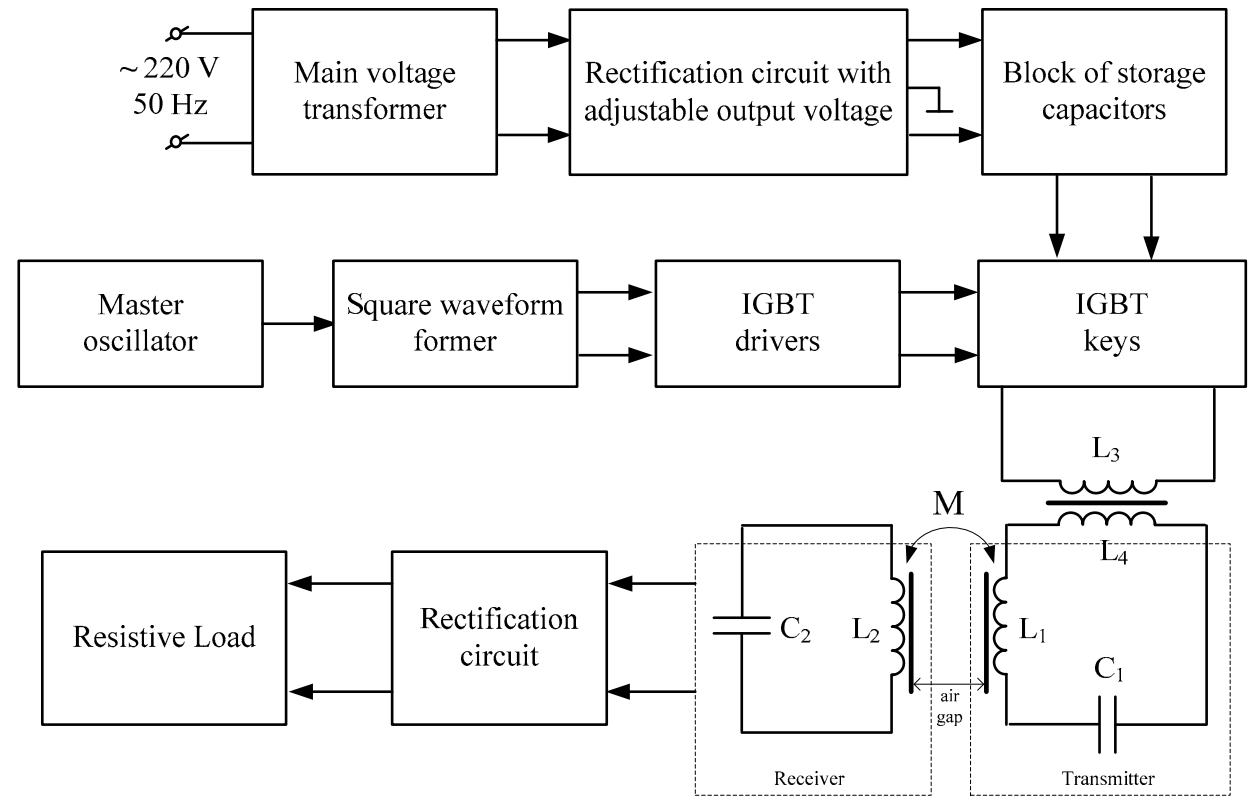

Figure 1. Block- diagram of the experimental installation of wireless power transfer

The experimental installation contains a transmitting circuit consisting of condenser $\boldsymbol{C}_{\boldsymbol{1}}$ and coil of inductance $\boldsymbol{L}_{1}$, and a receiving circuit consisting of coil of inductance $\boldsymbol{L}_{2}$ and condenser $\boldsymbol{C}_{2}$. Stimulation of the transmitting circuit is performed by the method of consecutive stimulation with the help of the stimulation transformer formed by the primary winding $\mathrm{L}_{3}$ and the secondary winding $\boldsymbol{L}_{\boldsymbol{4}}$.

The master oscillator forms right angle impulses which frequency may be regulated. Right angle impulses enter the generator of meander signals. The frequency of the meander signal is determined by the frequency of the right angle impulses. The meander signal enters the drivers of the IGBT transistors. The IGBT transistors' drivers are used to control the work of the IGBT transistors which perform the function of the electronic force keys. Usage of the IGBT transistors' drivers provides maximum coefficient of the IGBT transistors and their defense from the overloading. Performing the function of electronic keys, the IGBT transistors provide the discharge of accumulating condensers through the primary coil $\boldsymbol{L}_{3}$ of the stimulating transformer.

The rectifier of the net voltage is the source of the transmitted power. Constant voltage from the rectifier output charges the accumulating condensers. In the scheme of the net voltage rectifier there is 
provided adjustment of the amplitude of the rectified net voltage. It allows adjustment of the constant voltage at the rectifier output and, therefore, the condenser voltage. The voltage of the alternating current produced at the condenser $\boldsymbol{C}_{2}$, goes to the rectifying device of the receiving circuit. The output voltage of this rectifying device is the output voltage of the experimental installation of the wireless power transmission by the resonance induction method. The view of the developed experimental installation of the wireless power transmission is given in Fig. 2.

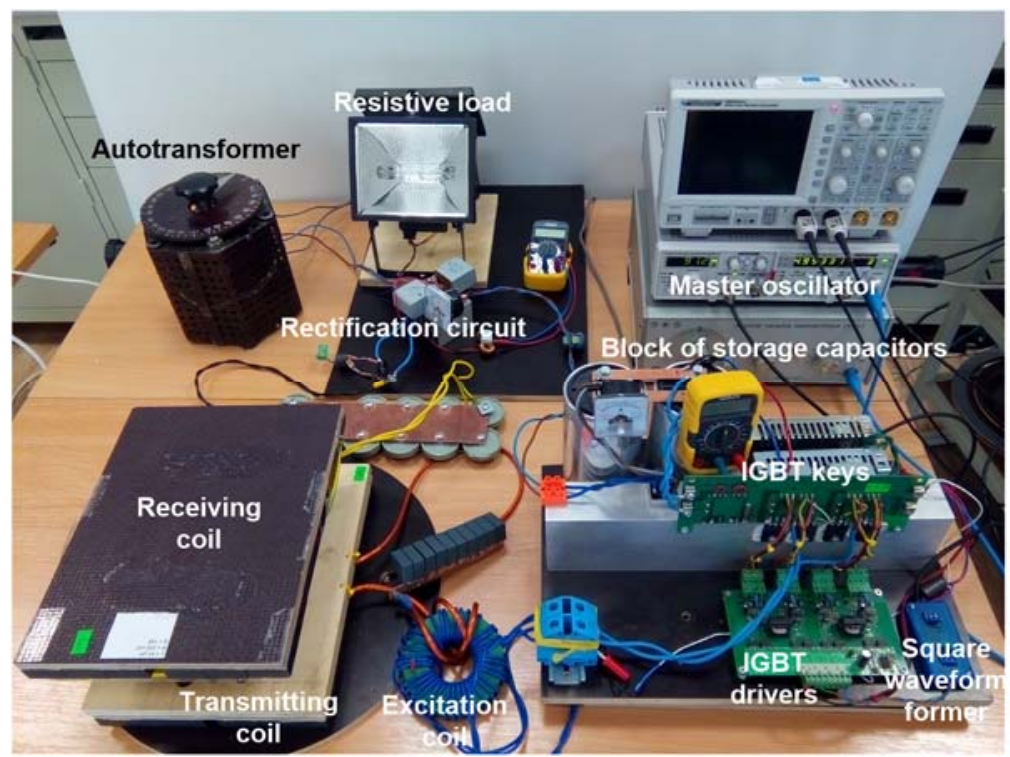

Figure 2. Experimental installation of the wireless power transfer

The auto transformer was used for adjusting the level of voltage produced at the bridge scheme of rectifying the net voltage. It provided adjustment of the constant voltage at the accumulating condensers (Fig.2.). As an active load of the rectification scheme at the receiving side there were used electric lighting lamps of different capacity. In the transmitting and receiving circuits there were used the rectangular DD coils of inductance.

The layout of the flat DD coil of inductance is given in Fig. 3. Fig. 3 shows the transmitting coil from the windings' side and Fig. 3.b shows it from the opposite side - from the side of the ferrite screen.

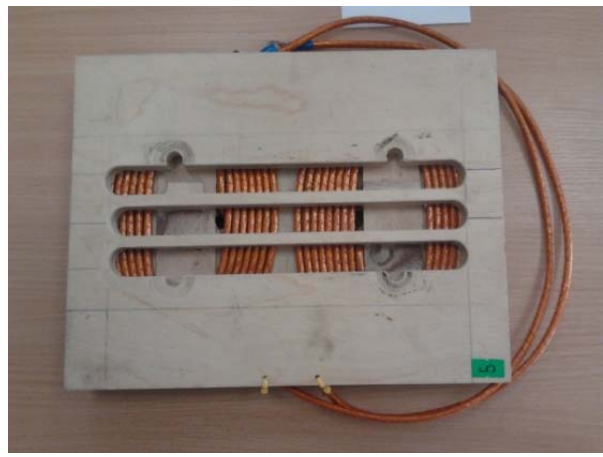

a

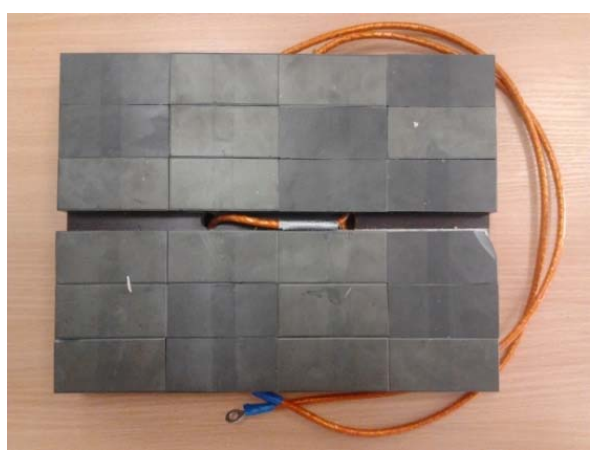

b

Figure 3. DD inductance coil (a - from the windings' side, b и d - from ferrite shield side)

Every spiral winding of the transmitting DD coil contains 8 windings of litz wire of the $45 \mathrm{~mm}$ diameter. Every spiral winding of the receiving DD coil contains 10 windings of the W-200-2L wire. The ferrite screen was made in the form a plate of ferrite magnet bars with the penetrability of $\mu=60$ and geometric sizes of $74 \times 35 \times 15 \mathrm{~mm}$. The squares of the transmitting and receiving DD coils were of the similar sizes: 290x230 mm. 


\section{Impact of the distance between the dd coils on the parameters of the resonance inductive system of the wireless power transfer}

With the wireless power transmission on the basis of the resonance mutual conduction of the circuits for the objects on move (in a dynamic mode), the distance between geometric centers of the transmitting and receiving coils may change. It changes because of the vertical and transversal shift of the receiving coil (moving object) regarding the center of the transmitting coil of the resonance transformer.

Change of the distance between the transmitting and receiving coils results in the change of the coefficient of mutual induction (mutual inductance) $\boldsymbol{M}$ and the coefficient of the kcB coils connection as well as in the change of the inductances of $\boldsymbol{L}_{\boldsymbol{1}}$ and $\boldsymbol{L}_{\boldsymbol{2}}$ coils of the transformer circuit. Change of the coefficient of the $\boldsymbol{k}_{\boldsymbol{c}}$ connection changes the reactive and active resistances in the oscillating circuits which brings about change of the quality, of the oscillating circuits' resonance frequency, of the AFC circuits and of the whole resonance transformer. As the result, there changes the transmitted capacity and the efficiency of the whole system of the wireless power transmission.

Affect of the distance between the DD coils inductance $\boldsymbol{d}$ on the parameters of transmitting coil of inductance was researched at different mutual dispositions of the DD inductance coils. For this, the geometric center of the receiving coil was shifted at a certain distance regarding the center of the transmitting coil in parallel to one of the sides of the transmitting coil or the receiving coil shifted regarding the transmitting coil at a certain angle. Table 1 shows the variants of the mutual disposition of the DD inductance coils. The figures show in green the overlapping parts of the mutually disposed DD inductance coils.

Table 1. Variants of the mutual disposition of the DD inductance coils

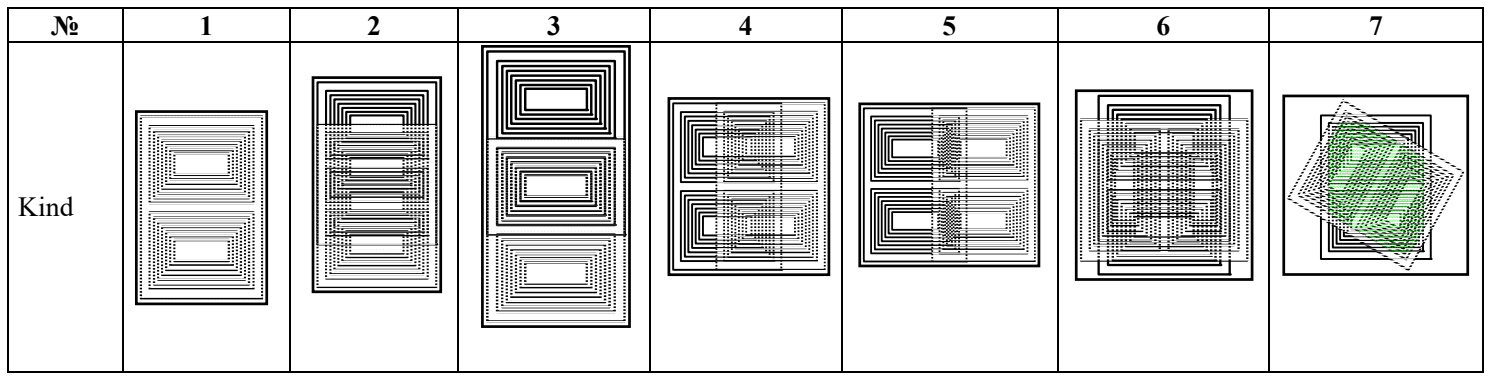

Fig. 4 shows the received dependencies of the connection coefficients between the ксви coils and the coefficients of the mutual inductance $\boldsymbol{M}$ for those variants of the mutual disposition of the DD coils at which comes into force mutual dependence of the transmitting and receiving coils.

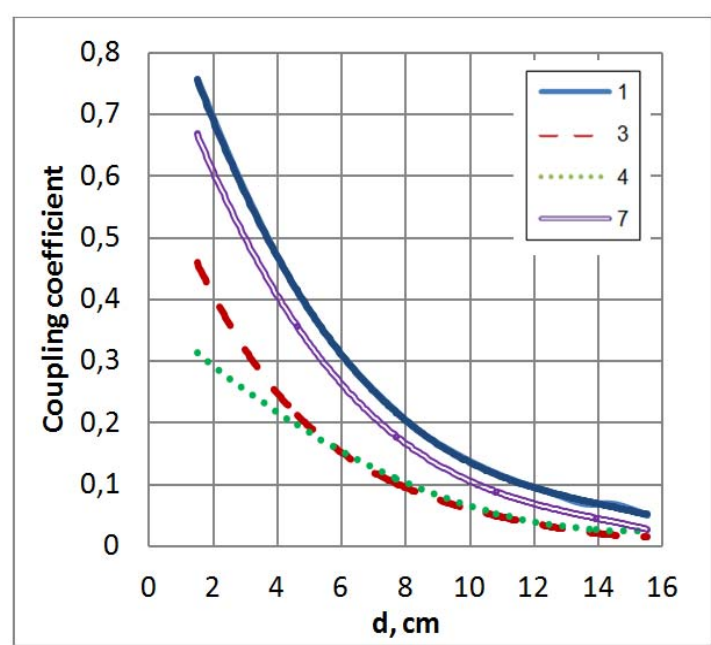

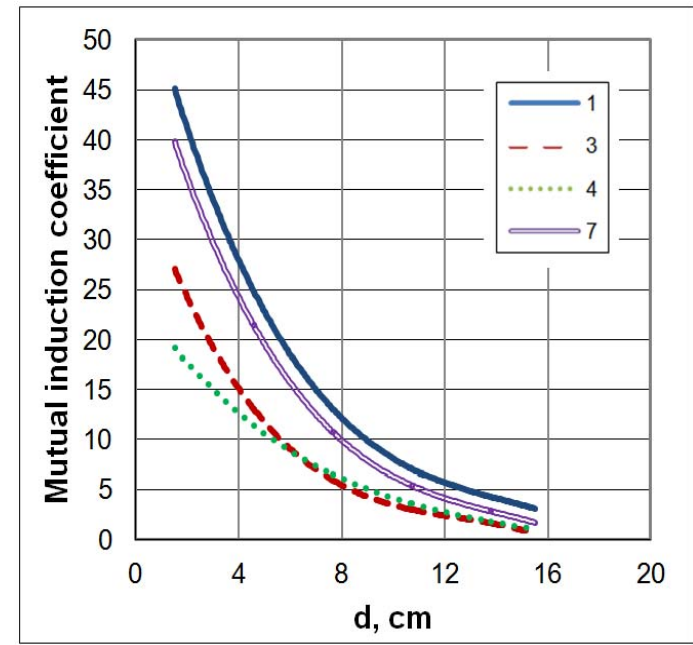

b

Figure 4. Influence of distance the DD inductance coil on on coupling coefficient different variants of f the mutual disposition of the DD inductance coils 
The values of the coefficient of connection and the coefficient of mutual induction are at maximum at any distance the DD inductance coils under the variant of the mutual DD coils disposition №1. In this variant of the DD coils disposition, the stream connection between the coils will be at maximum. The magnetic streams covering every winding of the DD coils will be turned one way [22]. Increase of the distance between the coils decreases the level of the magnetic field in the plane of the receiving coil. It brings about the decrease of the connection coefficient and the mutual induction coefficient.

If the DD coils of inductance are disposed in conformity with the variants 3,4 or 7 (table 1) the stream cohesion will be deceased but the one way direction of magnetic streams will remain. The disposition of the DD inductance coils, in conformity with the variants 2, 5,6 (Table 1) is characterized by the absence of the mutual connection: the coefficients of connection and the coefficients of mutual induction are close to zero. In such variants stream cohesion is decreased (variant №5), or the magnetic streams covering every DD coil winding will have different directions (variants 2 and 6).

The measurement scheme which block-diagram is shown in Fig.5 has been used for the study the power parameters of the resonant transformer. In this scheme series - parallel topology of the resonan transformer was used. The transmitting contour is a serial contour formed by the inductance $\boldsymbol{L}_{\boldsymbol{1}}$ and capacitor $\boldsymbol{C}_{1}$. The receiving contour is a parallel contour formed by the inductance $\boldsymbol{L}_{2}$ and capacitor $\boldsymbol{C}_{2}$.

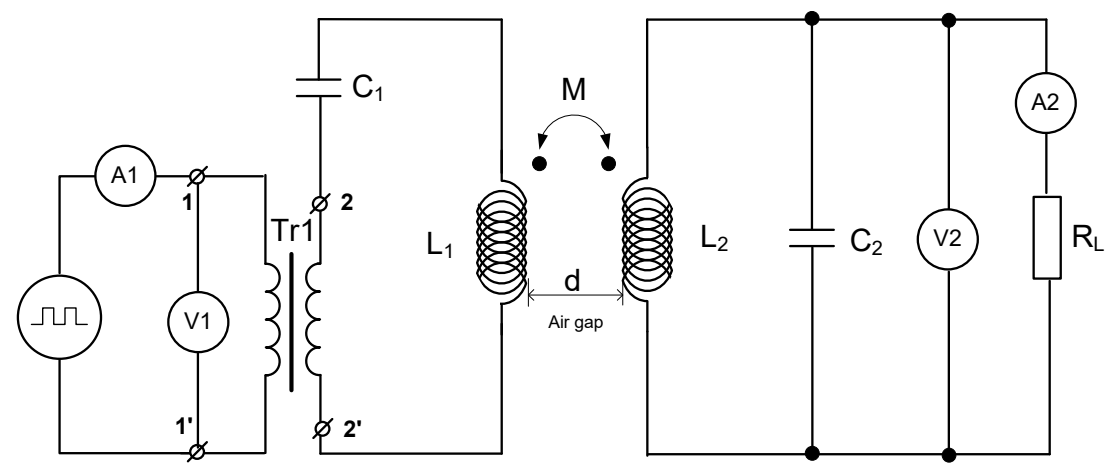

Figure 5. Measurement scheme for the study the power parameters of the resonant transformer

To excite oscillations in the transmitter contour transformer $\operatorname{Tr} 1$ has been used. Its secondary winding is included in the transmitting contour in series with $\boldsymbol{L}_{\boldsymbol{1}}$ and $\boldsymbol{C}_{\boldsymbol{l}}$. It provides a consistent inclusion of the exciting source of oscillations in the transmitting oscillatory contour. Transformer Tr1 has been made with toroidal core, its primary winding consisted of 18 turns and the secondary winding of the two turns. Load resistance RL is the input resistance of the rectification circuit (Fig. 2). Ammeters A1 and A2 were used to measure the input and output currents of the resonant transformer. Voltmeters V1 and V2 used to measure the voltage on the storage capacitor and on the load of resonant transformer.

The impact of air gap between DD coils has been investigated on parameters of the resonant transformer system of wireless power transfer. Investigations have been performed at two resonant frequencies: $\boldsymbol{f}_{\text {res }}=145 \mathrm{kHz}$ и $\boldsymbol{f}_{\text {res }}=85 \mathrm{kHz}$. The variant of the mutual arrangement of DD coils №1 (Table 1) was used. The distance between the DD coils $d$ was incremented by $3 \mathrm{~cm}$. The square wave signal frequency of the master oscillator (Fig. 1) was set equal to the resonant frequency of the resonant transformer.

Fig.6. shows the impact of the distance $d$ on the electrical parameters of the resonant transformer at a fixed voltage $\boldsymbol{U}_{\boldsymbol{c}}$ on the storage capacitor $\boldsymbol{U}_{\boldsymbol{c}}=50 \mathrm{~V}$ (Fig.1. and Fig.2.). We can see that the output current is maximal at distances dmax between DD coil of resonant contour. At these distances, the coupling coefficient between the coils is less than 0.1 (Fig.4.a): $\boldsymbol{d}_{\max }=9 \mathrm{~cm}$ when resonant frequency equals 145 $\mathrm{kHz}$ and $\boldsymbol{d}_{\max }=12 \mathrm{~cm}$ when resonant frequency equals $85 \mathrm{kHz}$. Further increase in distance d leads to decrease the output current, because it decreases the level of the magnetic field near the receiver coil, and decrease the coupling coefficient between the coils (Fig.4.a). Therefore the transmitting contour current are linearly depends on $d$, except on the edge areas. The current value is small in the initial areas, due to the large coupling coefficient (the coefficient of mutual induction (Fig.4.a and Fig.4.b). The transmitting contour current reaches its maximum value with increasing the distance $d$, because the coupling is practically nonexistent between the contour coil $\left(\boldsymbol{k}_{\boldsymbol{c}}<0.05\right.$ at Fig.4.a).The output current decrease when the distance in the initial area due to decrease of transmitting contour current. 

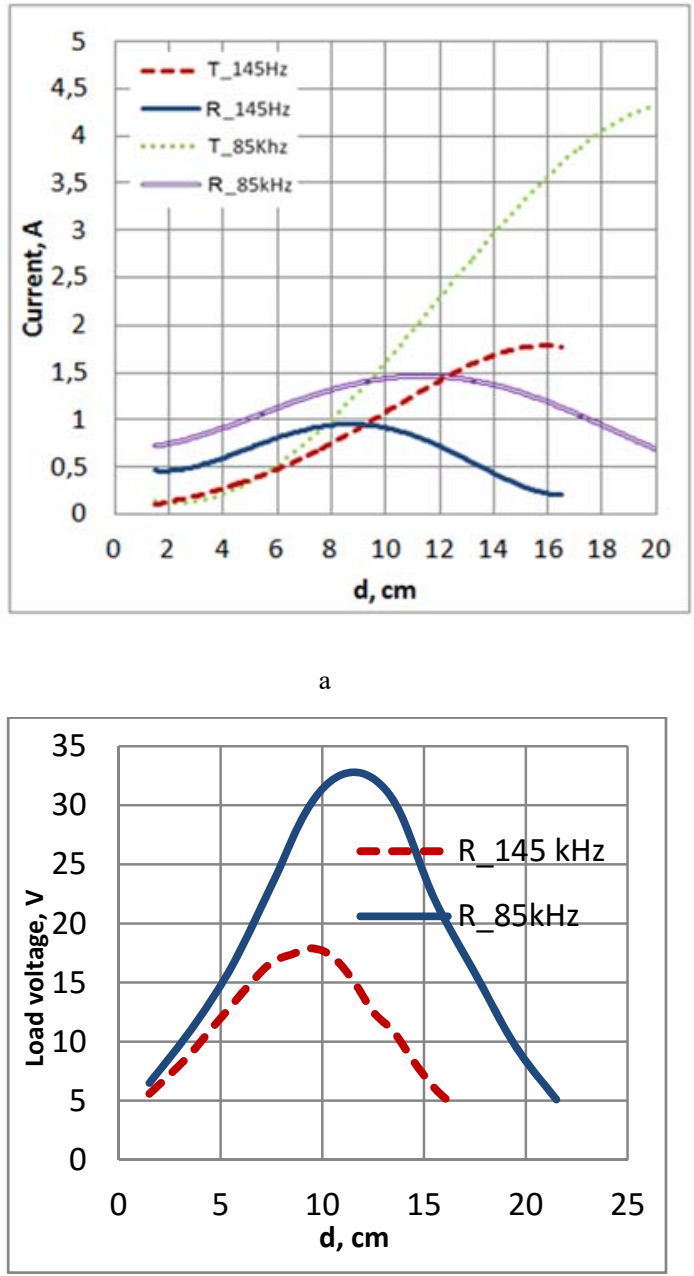

$\mathrm{c}$
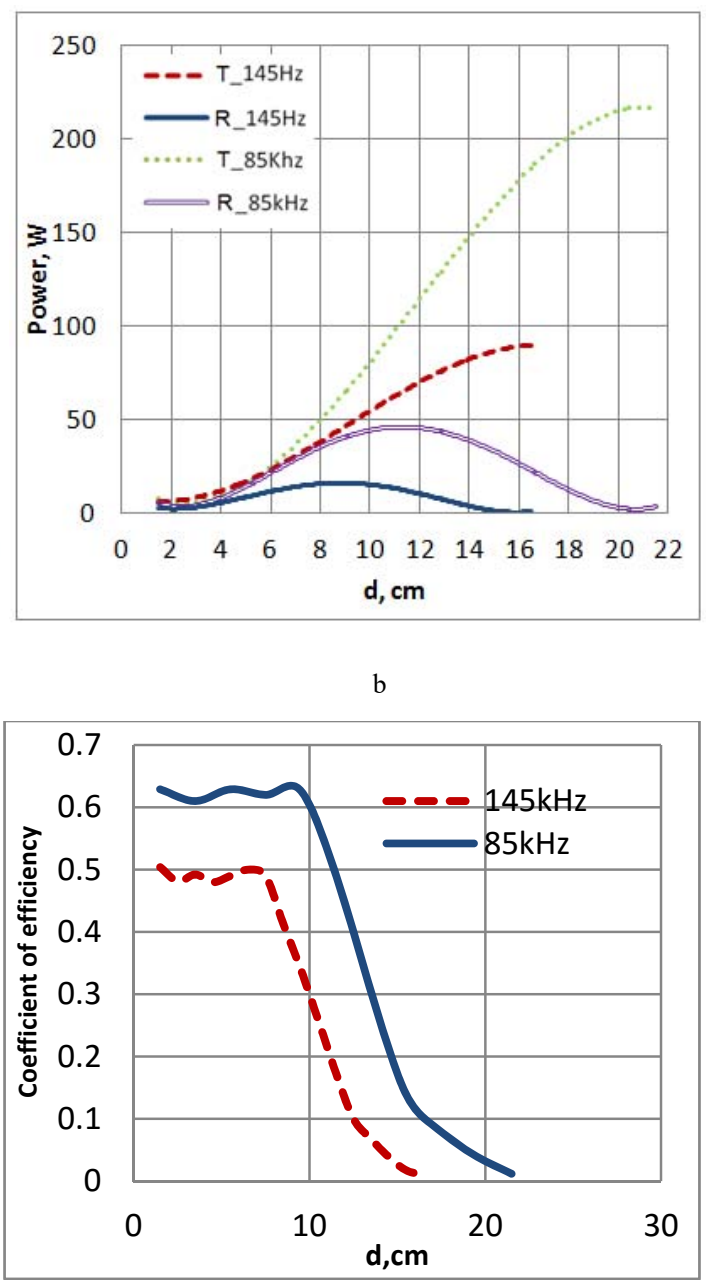

d

Figure 6. The impact of the distance $\mathrm{d}$ on the electrical parameters of the resonant transformer with $\mathrm{Uc}=50 \mathrm{~V}$ and two resonant frequencies ( $a-$ on the input and output currents; b - on the input and output powers; $c$ - on voltage at the load receiving circuit; d- on power transmission coefficient; T-input parameters; R- output parameters)

The load resistance RL is equal $18 \mathrm{ohms}$, and it determines the f voltage at the load of the receiving contour (Fig.6.c). The kind of these dependences corresponds of output current changes. The kind of the output power dependences (Fig.6.b) is determined by the dependence of the transformer output current. All three output dependences have extremes but when resonant frequency equals $85 \mathrm{kHz}$ dependences have maximums at greater distance between the coils. Input power (Fig.6.b) varies as well as the input current by increasing the distance $\boldsymbol{d}$ (Fig.6.a).

Fig. 6.d demonstrates the dependences of power transfer coefficient (рис.10.b) from the distance $\boldsymbol{d}$. When $\boldsymbol{d}>\boldsymbol{d}_{\max }$ power transfer coefficients diminish rapidly, as at such distances there is a simultaneous increase input power, because increasing the input current with decreasing output power due to the reduction of the output current. Dependences of the power transfer coefficient do not change at the beginning of interval, since the speed of increase output and input power is the same with increasing distance $\boldsymbol{d}$.

The resonant frequency of coupled contours decreases at changing the distance $\boldsymbol{d}$. Therefore, during research of dependencies which are shown in Fig. 6, values of the resonant transformer resonant frequency were fixed at each distance $\boldsymbol{d}$. The resonance frequency was determinated by sweeping the frequency of the master oscillator (Fig.1) to obtain the maximum load voltage in the receiving contour. Fig.7. shows the impact of the distance $\boldsymbol{d}$ on the resonant frequency of the resonant transformer at a fixed voltage on the storage capacitor $\boldsymbol{U}_{\boldsymbol{c}}=50 \mathrm{~V}$.

It is seen that the resonant frequency of the resonant transformer decreases with increasing distance $\boldsymbol{d}$. It's value becomes equal to the resonance frequency of the transmitting contour, if the distance $\boldsymbol{d}$ is equal 
dmax. However, further increase in $\boldsymbol{d}$ (decrease $\boldsymbol{k}_{\boldsymbol{c}}$ ) causes a slight increase in the resonant frequency to several hundred Hz. For large distances, the current in the transmitting contour increases to several amperes (Fig.6.a) that leads to increasing the temperature of the winding of the transmitting coil. Fig.8. shows the change of transmitting coil temperature when the distance between coils changes with $\boldsymbol{U}_{\boldsymbol{c}}=50$. When $\boldsymbol{d}>$ $\boldsymbol{d}_{\max }$ the transmitting coil temperature increases linearly. In result the inductance of the resonance transformer transmitting coil decrease, and the resonant frequency of the resonant transformer increase (Fig.7).

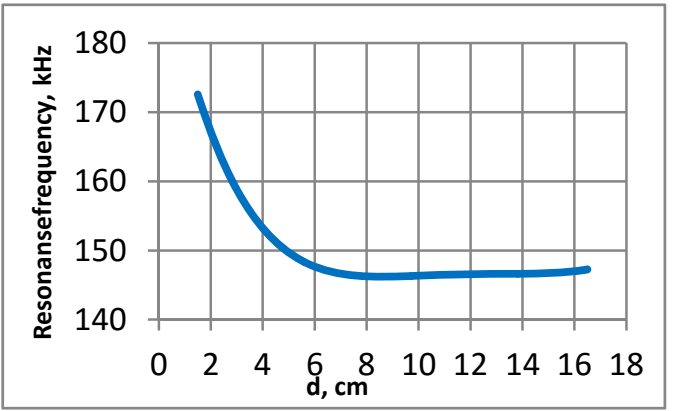

a

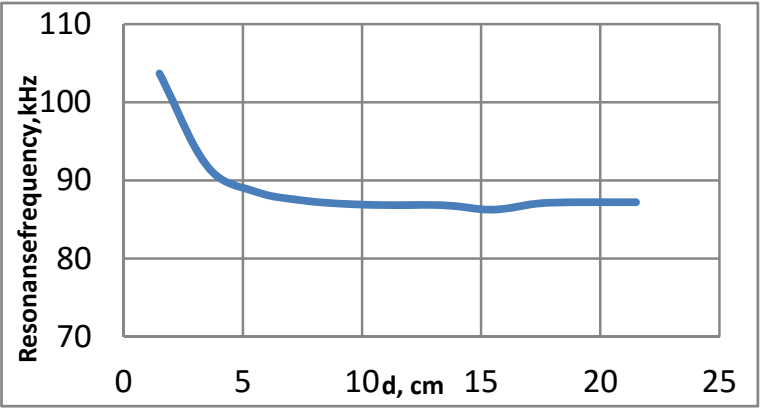

b

Figure 7. Impact of the distance between the coils on the resonant frequency of the transformer with $\mathrm{U}_{\mathrm{c}}=50 \mathrm{~V}$

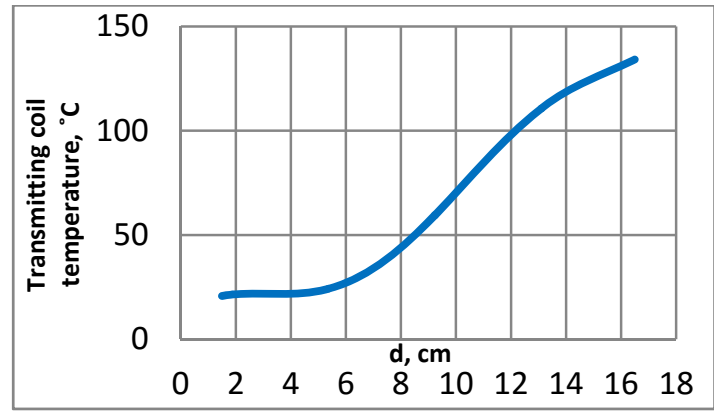

a

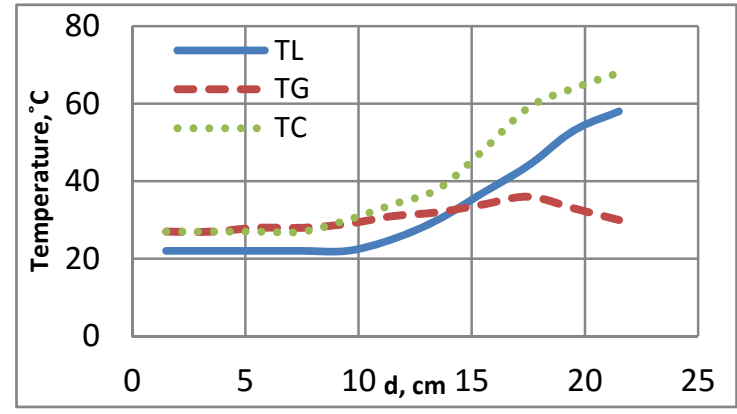

b

Figure 8. Temperature changes of transmitting coil $\left(\mathrm{a}-f_{\text {rez }}=145 \mathrm{kHz}\right)$ and of transmitting part structural elements of resonant transformer $\left(\mathrm{b}-f_{\text {rez }}=85 \mathrm{kHz}\right)$ when the distance between the inductance coils is changed with $\mathrm{U}_{\mathrm{c}}=50 \mathrm{~V}$

Fig. 8.b show temperature changes of structural elements that impact on the resonant frequency: transmitting contour DD coil (TL), the transmitting contour capacitor (TC) and IGBT transistors (TG). These curves were obtained by air-cooling of those components. The temperature TG has changed slightly when the distance between the coils increases. It's clear that temperature of the transmitter coil and the temperature pf capacitor is linearly increased if $\boldsymbol{d}>10 \mathrm{~cm}$. Capacitors used in these experiments had a high thermal stability, therefore their capacitance changed slightly. Increasing the temperature of the transmitting DD coil give a decrease of its inductance and increase the resonant frequency of the resonant transformer when $\boldsymbol{d}>12 \mathrm{~cm}$, even with air cooling.

\section{Impact impact of voltage of storage capacitor on the eficiency of the wireless power transfer with the employment resonant inductive method}

To transmit the required power to the load of resonant inductive transformer, the input voltage of the resonant transformer must be adjusted. The impact of the storage capacitor voltage Uc has been investigated on the frequency and energy parameters of the resonant transformer system of wireless power transfer. Investigations have been performed at two resonant frequencies: $\boldsymbol{f}_{\text {res }}=145 \mathrm{kHz}$ и $\boldsymbol{f}_{\text {res }}=85 \mathrm{kHz}$. Distances between DD coils of the transformer are equal to dmax: $\boldsymbol{d}_{\boldsymbol{m a x}}=9 \mathrm{~cm}$ when $\boldsymbol{f}_{\text {res }}=145 \mathrm{kHz}$ and $\boldsymbol{d}_{\boldsymbol{m a x}}=12$ when $\boldsymbol{f}_{\text {res }}=85 \mathrm{kHz}$. Fig. 9 and Fig. 10 demonstrates the results of investigations.

The storage capacitor voltage Uc was varied from $10 \mathrm{~V}$ to $170 \mathrm{~V}$. Voltage boost of the storage capacitor Uc increases the transmitting oscillation circuit current (input current - T) and load current (output current - R). These dependencies have a small non-linearity (Fig.9.a). 

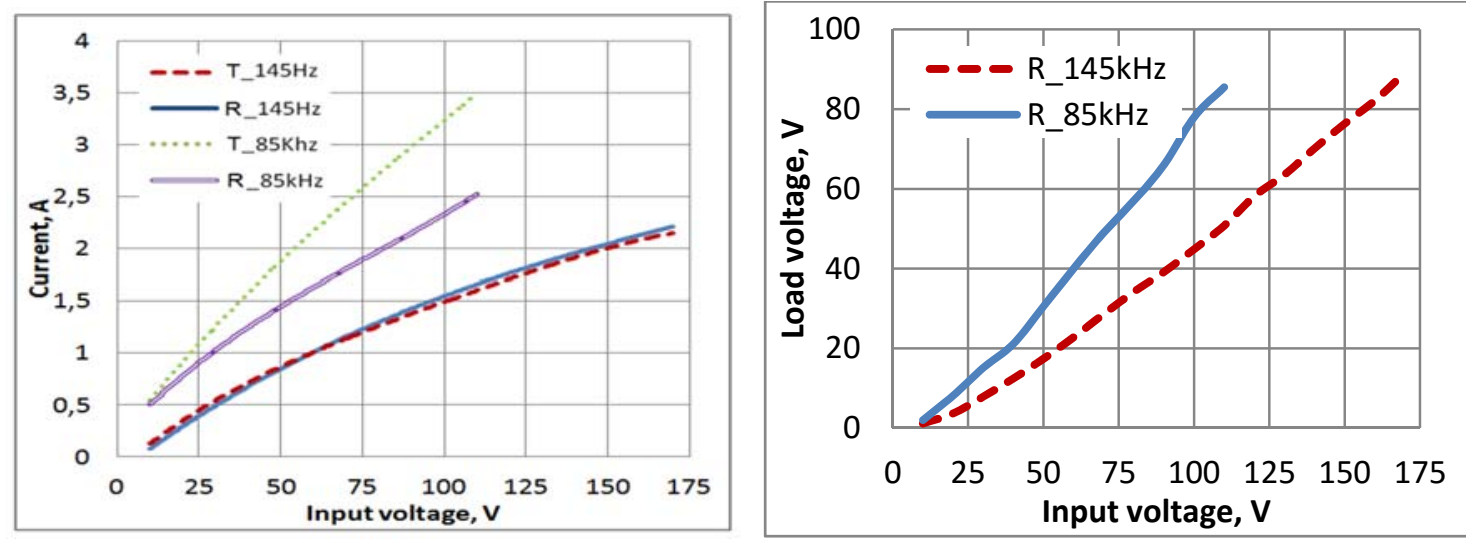

a
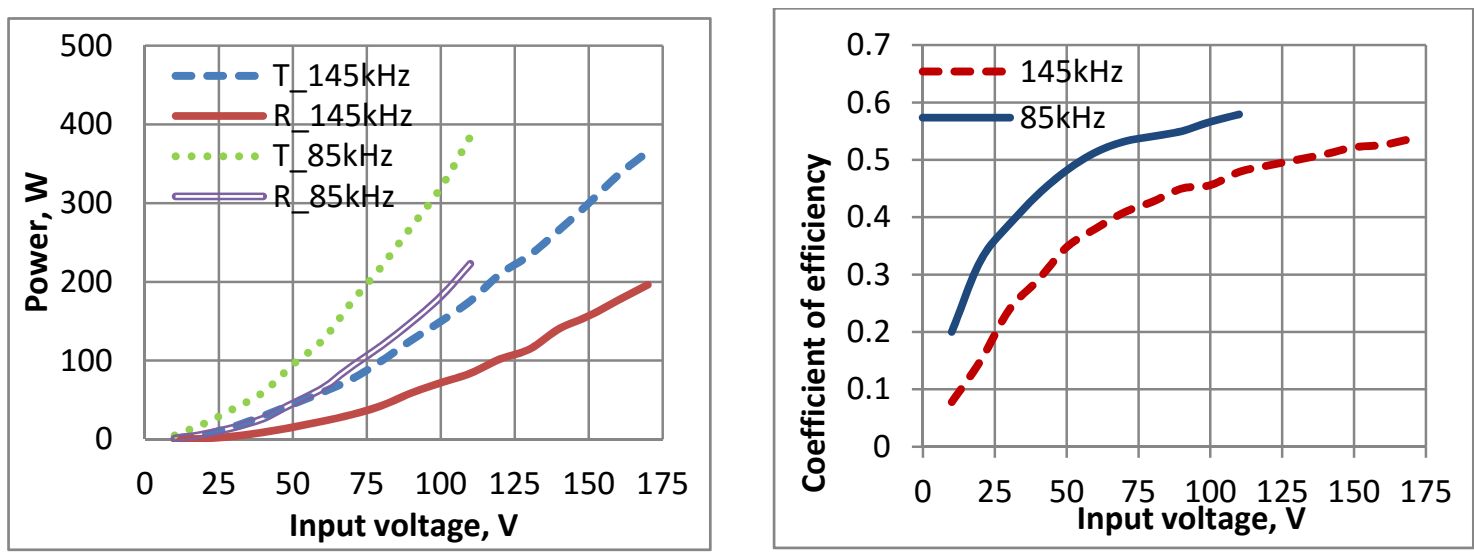

c

d

Figure 9. Impact of voltage $U_{c}$ on the characteristics of the resonant system for $\mathrm{f}_{\text {rez }}=145 \mathrm{kHz}$ and $\mathrm{f}_{\text {rez }}=85 \mathrm{kHz}$ when $\mathrm{d}=\mathrm{d}_{\text {max }}$ $(\mathrm{a}-$ on currents in contours; $\mathrm{b}$ - on load voltage; $\mathrm{c}$ - on input and output power; $\mathrm{d}$ - on power transmission efficiency; $\mathrm{T}$ - transmitting contour; $\mathrm{R}$ - receiving contour)

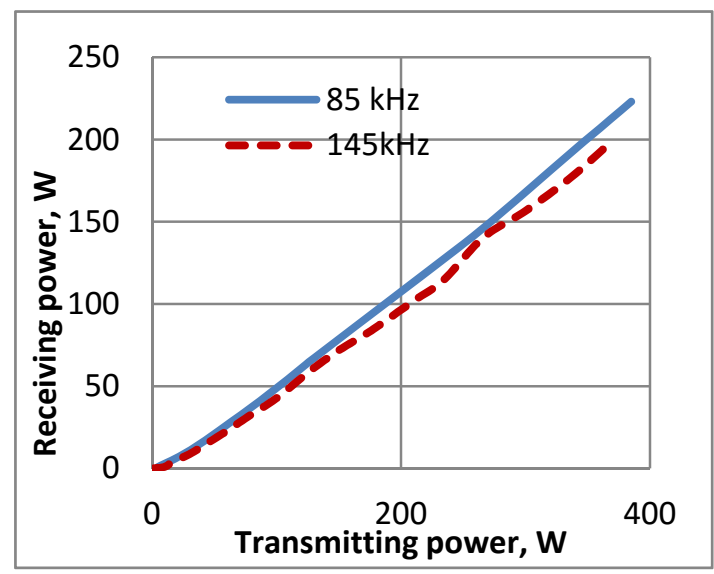

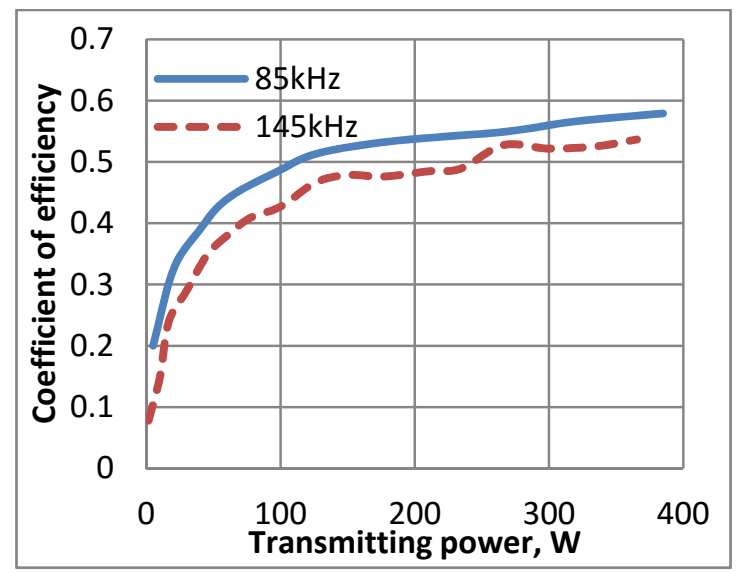

b

Figure 10. Eficiency of the wireless power transfer with the employment of DD inductance coils on resonanse frequencies $\mathrm{f}_{\text {res }}=145 \mathrm{kHz}$ and $\mathrm{f}_{\text {rez }}=85 \mathrm{kHz}$ when $\mathrm{d}=\mathrm{d}_{\text {max }}(\mathrm{a}$ - the dependences receiving power of transmitting power; $\mathrm{b}$ - the dependences power efficiency of transmitting power)

The voltage at the load of receiving oscillation circuit depends on the voltage Uc weak nonlinear (Fig.9.b). This non-linearity is due to increased impedance of lighting lamps, which are load of circuit rectification (Fig.1). When the voltage $\boldsymbol{U}_{\boldsymbol{c}}$ increases, the output current growth slows and lighting lamps 
impedance continues to increase. This leads to a linearization of the output voltage dependence. The input power and the output power depend quadratically on the voltage $\boldsymbol{U}_{\boldsymbol{c}}$ (Fig.9.c). Power transfer coefficient is defined as the ratio of output power to transmit power. When the voltage $\boldsymbol{U}_{\boldsymbol{c}}$ increases power transfer coefficient increases as well. However, its growth is slowing at a voltage of $\boldsymbol{U}_{\boldsymbol{c}}$, exceeding $50 \mathrm{~V}$ (Fig.9.d).

Figure 10 demonstrates the dependences of output power of resonant transformer (Fig.10.a) and dependences of power transfer coefficient (рис.10.b) from power transfer. These dependences characterize the power transfer efficiency of resonant inductive method with the employment of DD induction coils. It is seen that when distances between DD coils is equal to optimal the output power depends on the transmitted power almost linearly (рис.10.a). However, the power transfer efficiency is small, if the input power is less than $50 \mathrm{~W}$, though its changes are essential. If the input power exceeds $100 \mathrm{~W}$, power transfer coefficients increases slightly, and they tend to the value 0.6 . These results show that the power transfer by a resonance method with the employment of DD induction coils is more effective when the resonant frequency is $85 \mathrm{kHz}$. (Fig.9.d and Fig.10).
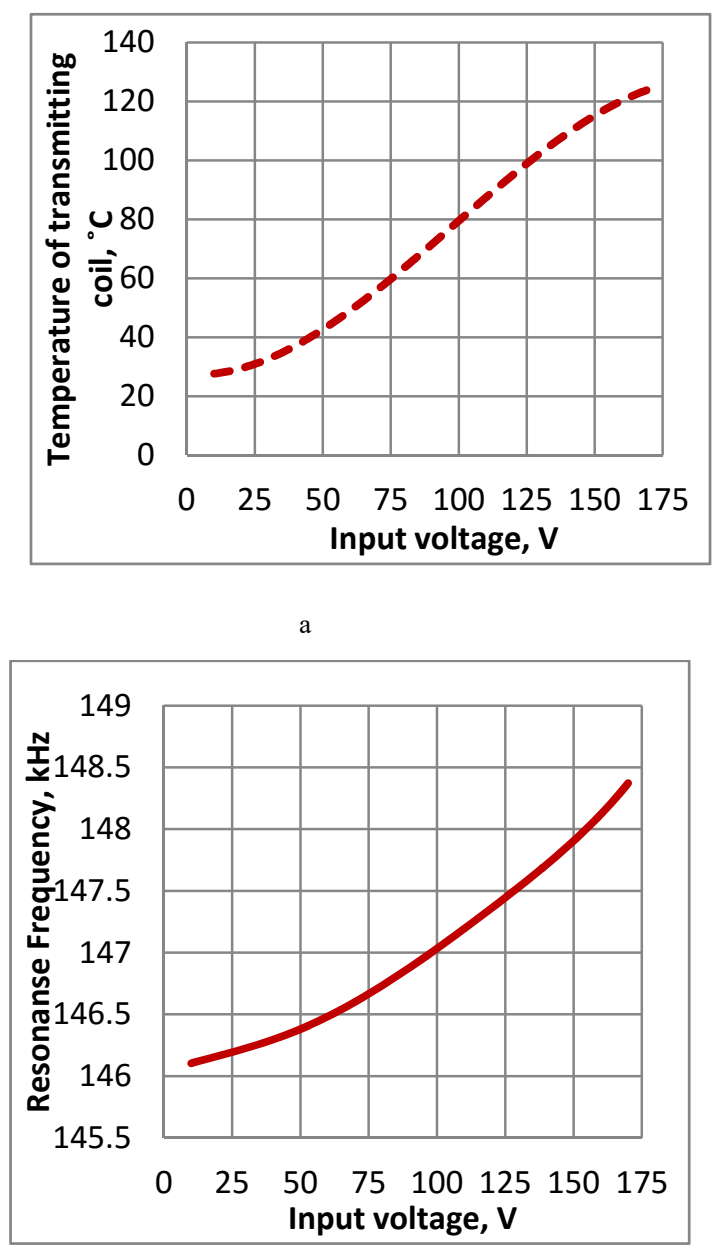

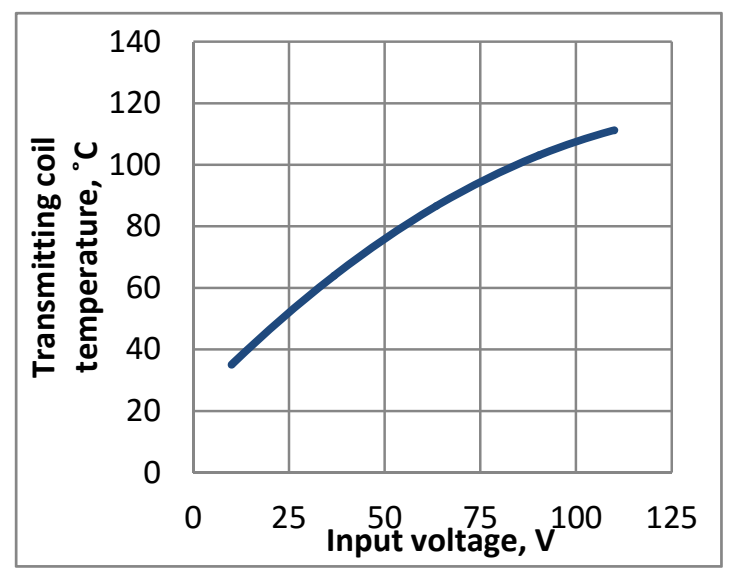

$\mathrm{b}$

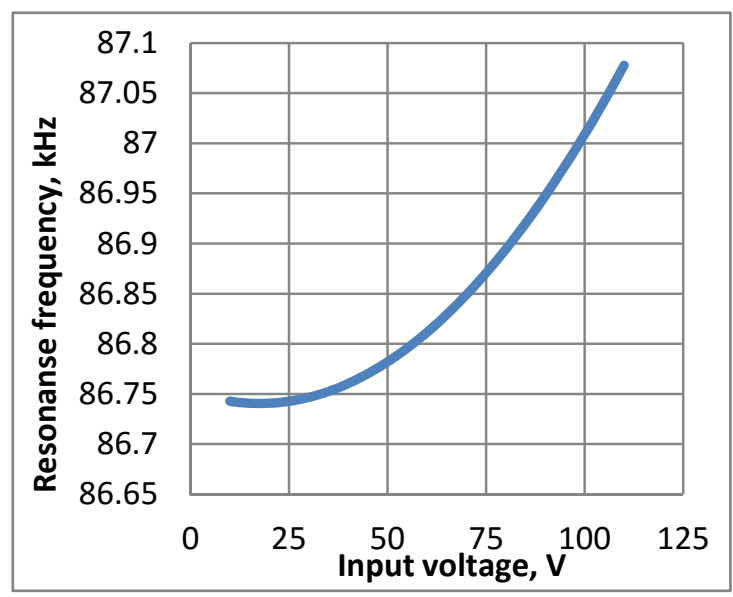

$\mathrm{d}$

Figure 11. Impact of voltage $\mathrm{U}_{\mathrm{c}}$ on temparature of tranmiting coil (a and b) and on resonant frerquency of resonant transformer (c and d) with $d=d_{\max }$ for resonanse frequencies $f_{\text {res }}=145 \mathrm{kHz}$ (a and c), $f_{\text {res }}=85 \mathrm{kHz}$ (b and d)

High current in the transmitting contour (Figure 9) causes the heating of DD transmitting coil to temperatures close to the maximum allowable temperature of litz wire $\left(130^{\circ}\right)$, which was used for the windings of the transmitter coil (Fig.11.a and Fig.11.b .). The transmitting DD coil inductance decreases at such high temperatures. So when storage capacitor voltage Uc was increased the resonant frequency of resonant transformer also increased (Fig.11.с и Fig.11.d). The resonant frequency of resonant transformer depends quadratically on the storage capacitor voltage $\boldsymbol{U}_{\boldsymbol{c}}$ (Fig.11.c), and it changes significantly, especially when $\boldsymbol{f}_{\text {res }}=145 \mathrm{kHz}$.

Analysis of the dependences on figure 6, figure 9 and figure 10 reveals that power transfer coefficients do not exceed 0.6 , ie the use of DD coils do not provide high efficiency of wireless power 
transfer. To increase the transmitted power in the load and the power transfer coefficient to values close to 1 can increase the factor of transformation (increasing the number of windings in the secondary winding) excitation transformer Tr1 (Fig. 5.). This provides an increase the current in the transmitting coil of resonant transformer and an increase of transmitted power. At the same time the transmitting DD coil $\boldsymbol{L}_{1}$ temperature will be higher. The temperature stability of this coil was unable to provide during our research, unfortunately.

\section{Research efficiency of wireless power transfer without resonance properties on the receiving side}

An important condition for high efficiency of resonant inductive method of wireless power transfer is equal resonance frequencies at the transmitting and receiving oscillatory contours of resonant transformer. However, the investigation results show that the resonant frequency of the transmitting resonant contour of the transformer depends on the distance $d$ (the distance between the DD coils), and the heating temperature of transmitter coil (Fig.7, Fig.8, Fig.11). Therefore, it is necessary to eliminate or to decrease an impact of above factors to provide high efficiency of power transfer with the employment of DD induction coils. The following methods can be used for this:

- the thermostabilization of the electronic components of resonant transformer;

- an automatic adjustment of the resonant frequency of the transmitting contour of resonant transformer at a fixed frequency master generator;

- an automatic tuning of master generator at the resonant frequency of the transmitting contour of the resonant transformer.

The third method allows simplifying the requirements for thermal stability and thermostabilization of the electronic components of resonant of the transformer and the resonant circuit is not used at the receiving side of resonant transformer. For example, be used on the receiving side receiving DD inductance $\boldsymbol{L}_{2}$ as the secondary winding of the transformer only by removing the contour capacitor $\boldsymbol{C}_{2}$. Fig. 12, Fig. 13 and Fig.14 demonstrate the investigation results of inductive resonant method of wireless power transfer with the employment of a transformer circuit at the receiving side. Investigations have been performed at the transmitting contour resonant frequency $\boldsymbol{f}_{\text {res }}=85 \mathrm{kHz}$.

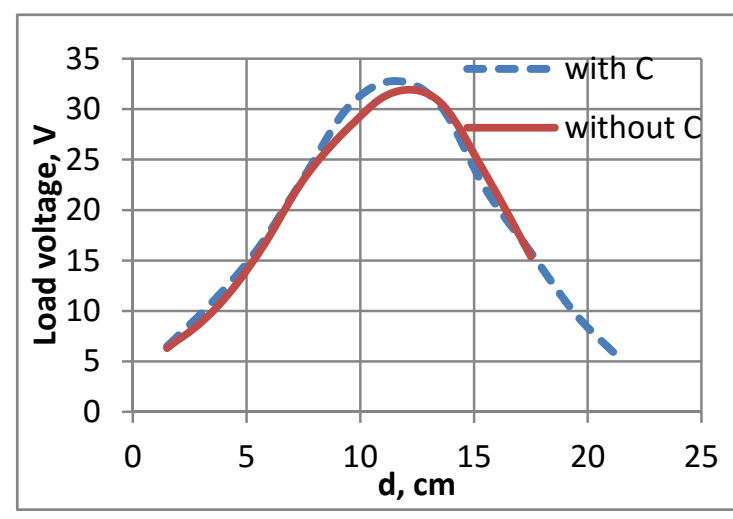

a

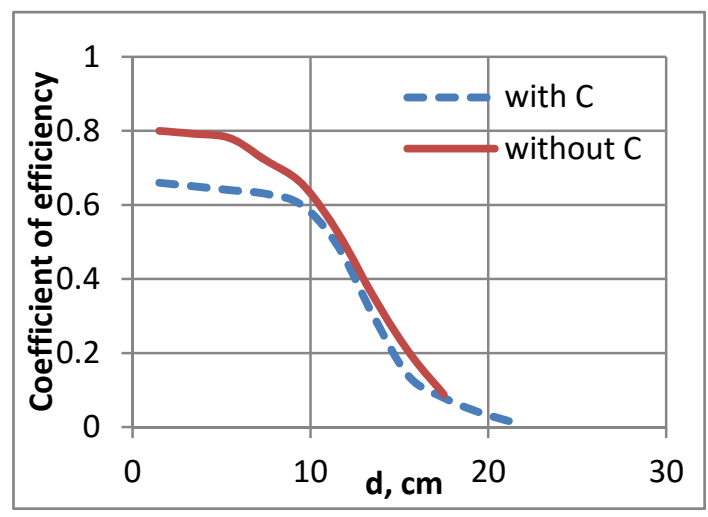

b

Figure 12. Impact of distance between the DD coils on the electrical parameters when the capacitor $\mathrm{C}_{2}$ is connected and absent on the receiving side for the transmitting frequency of the resonance circuit $f_{\text {res }}=85 \mathrm{kHz}$ and $U_{c}=50 \mathrm{~V}$ (a- on load voltage of receiving contour; $b$ - on coefficient of power efficiency)

Figure 12.a demonstrates the dependences of load voltage on the receiving side of transformer without capacitor $\boldsymbol{C}_{2}$ and with capacitor $\boldsymbol{C}_{2}$. When the capacitor is absent, load voltage extremum decreases and shifts slightly. Fig.12.b demonstrates the dependences of the power transfer coefficient from distance between DD coils. It is seen that the distance $d$ are equally influences on the power transfer coefficient as with a capacitor $\boldsymbol{C}_{2}$ and without capacitor $\boldsymbol{C}_{2}$, but the values of the transmission power coefficient greater when disconnecting a capacitor $\boldsymbol{C}_{2}$.

The output power depends quadratically from the voltage $\boldsymbol{U}_{\boldsymbol{c}}$ both with the capacitor $\boldsymbol{C}_{2}$ and without the capacitor $\boldsymbol{C}_{2}$ at the receiving side of resonant transformer. However, when no resonance properties on the receiving side the steepness of the output power more. This is because the load voltage and load current 
of resonant transformer increase simultaneously. Since the output power is higher for any $\boldsymbol{U}_{\boldsymbol{c}}$ in the absence of contour capacitor $\boldsymbol{C}_{2}$, so increasing of voltage $\boldsymbol{U}_{\boldsymbol{c}}$ leads to a growth of the power transfer coefficient (Fig. 13.b).

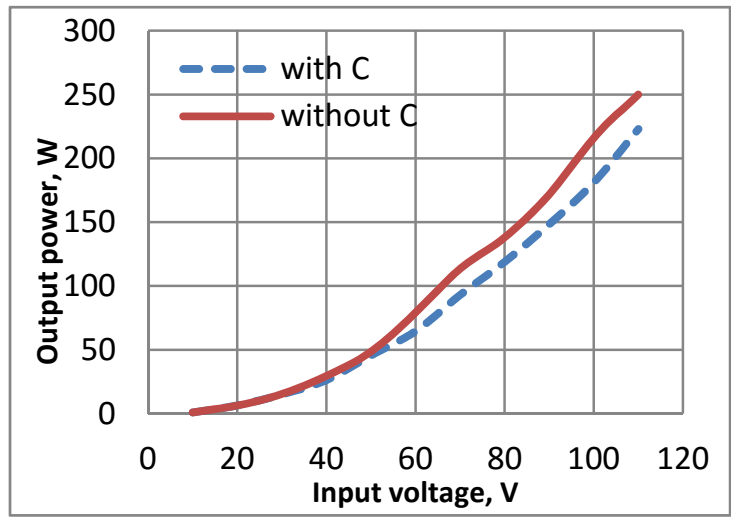

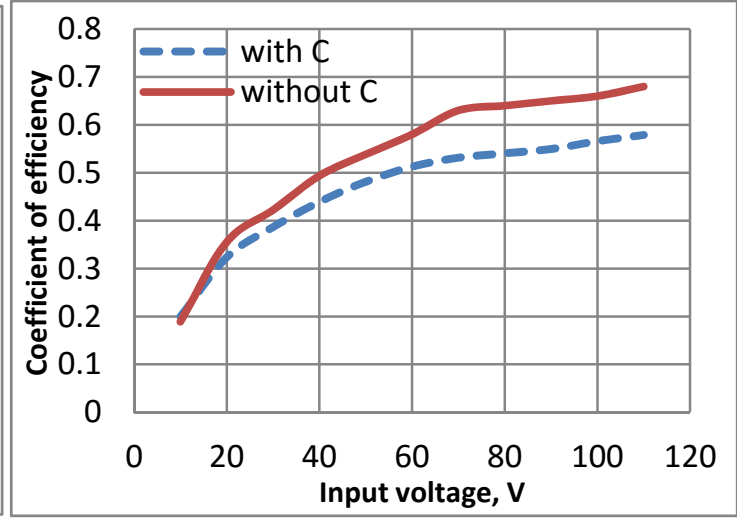

b

Figure 13. Impact of voltage $U_{c}$ on the efficiency of power when the capacitor $\mathrm{C}_{2}$ is connected and absent on the receiving side for the transmitting frequency of the resonance circuit $f_{\text {res }}=85 \mathrm{kHz}$ and $\mathrm{U}_{\mathrm{c}}=50 \mathrm{~V}(\mathrm{a}-$ on output power; $\mathrm{b}-$ on coefficient of power efficiency)

Figure 14 demonstrates the dependences of output power of resonant transformer (Fig.14.a) and dependences of power transfer coefficient (Fig.14.b) from power transfer with the employment of DD induction coils when contour capacitor $\boldsymbol{C}_{2}$ is absent on the receiving side (receiving contour has no the resonance properties) and when contour capacitor $\boldsymbol{C}_{2}$ is connected to the receiving coil $L_{2}$ of resonant transformer. It is seen that the output power depends on the transmitted power almost linearly in both cases, but contour capacitor $\boldsymbol{C}_{2}$ was disconnected the dependence of the output power has a greater steepness. Therefore, power transfer coefficient has larger magnitude when contour capacitor $\boldsymbol{C}_{2}$ is not on the receiving side at any values of the input power (Fig.14.b).

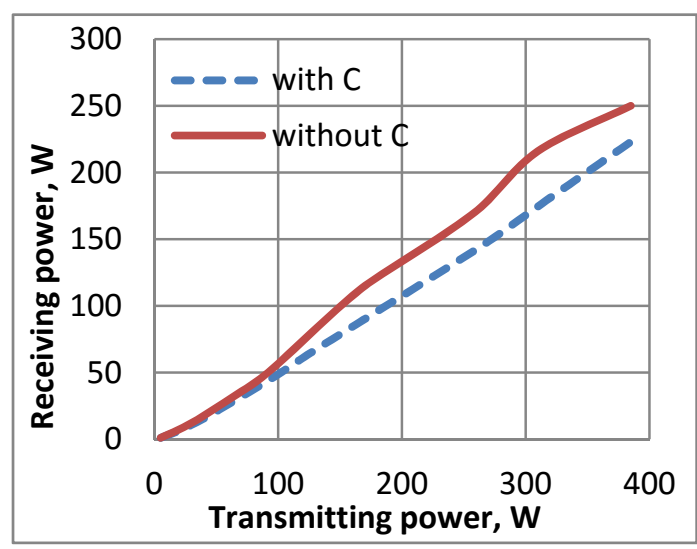

a

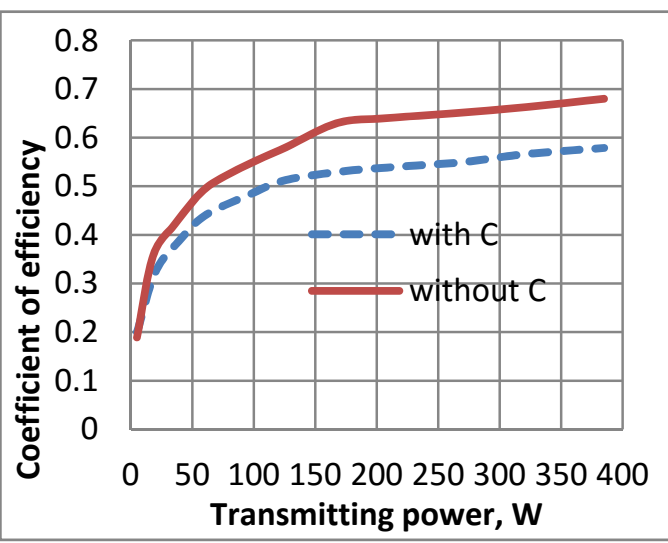

b

Figure 14. Efficiency of power transfer with $\mathrm{d}=12 \mathrm{~cm}$ between DD inductance coils when the contour capacitor is disconnected on the receiving side ( $\mathrm{a}$ - the dependence of receiving power to transmitting power; $\mathrm{b}$ - the dependence of coefficient of power efficiency to transmitting power)

The research results are presented in Fig.12, Fig.13 and Fig.14 show that the efficiency of the wireless power transfer with the employment of DD induction coils is higher when the receiving side of resonant transformer has not the resonance properties. This is because the amplitude frequency characteristic of resonant transformer is determined by the amplitude frequency characteristic of the transmitting contour (by its resonance frequency and quality factor) when contour capacitor $\boldsymbol{C}_{2}$ is not on the receiving side: and Q factor. 


\section{Conclusions}

The results of the above research allow making the following conclusions:

- for the researched DD coils with the length of the bigger side $290 \mathrm{~mm}$ the coefficient of connection is less than 0,1 (sign of any weak connection) at distances more than $12 \mathrm{~cm}$ for any mutual position of the transmitting and receiving coils;

- increasing the distance between the transmitting and receiving DD coils results in the decreasing the resonance frequency of the transformer which aims at the resonance frequency of the transmitting inductance coil;

- effective wireless transmission of power with the use of the DD inductance coils of the resonance transformer is provided under the optimal distance between them;

- decreasing the resonance frequency of the transmitting circuit increases the efficiency of the wireless power transmission and increases the optimal distance between the coils of the resonance transformer;

- absence of resonance properties at the receiving side allows increasing the capacity of the load and the coefficient of the capacity transmission;

- increase of the coefficient of the capacity transmission up to the values close to 1 can be provided by increasing the coefficient of transformation of the stimulating transformer and the temperature stability of the parameters of the transmitting DD inductance coil;

- to use the DD inductance coil for the wireless charge of the automobile accumulator on move with the account of the modern transport means clearance it is required to provide the following: parameters of the DD inductance coils, the level of capacity of the transmitted power source, the level of capacity produced in the transmitting circuit of the resonance transformer, the temperature stability of the constructive elements of the transmitting circuit.

\section{Acknowledgements}

This paper has been published within the research project 'Research on dynamic wireless charging of electric vehicles and development of experimental model' was carried out within grant program by European Regional Development Fund for general industrial research and for projects dealing with new product and technology developments. Latvian Investment and development agency Contract number: L-KC-11-0002 project number: KC/2.1.2.1.1/10/01/008.

\section{References}

1. Budhia, M., J. Boys, T., G., Covic, A. and Chang-Yu, H. (2013) Development of a single-sided flux magnetic coupler for electric vehicle IPT charging systems. IEEE Trans. Ind. Electron., vol. 60, no. 1, pp. 318-328.

2. Covic, G. and Boys, J. (2013) Modern Trends in Inductive Power Transfer for Transportation Applications. IEEE journal of emerging and selected topics in power electronics, vol. 1, no. 1, pp. 3848.

3. Grajski, K. A., Tseng, R. and Wheatley, C. (2012) Qualcomm Incorporated. Loosely-Coupled Wireless Power Transfer: Physics, Circuits, Standards. - IEEE.

4. Kurs, A., Karalis, A., Moffatt, R., Joannopoulos, J. D., Fisher, P. and Soljačić, M. (2007) Wireless Power Transfer via Strongly Coupled Magnetic Resonances. Science: Vol. 317 no. 5834 pp. 83-86.

5. Li, S. and Mi, C. (2015) Wireless power transfer for electric vehicle applications. IEEE journal of emerging and selected topics in power electronics, vol. 3, no. 1, pp.4-17.

6. Miller, J., White, C. P., Onar, O. C. and Ryan, P. M. (2012) Grid side regulation of wireless power charging of pulg-in electric vehicles. IEEE Energy Conversion Congress and Exposition (ECCE), pp. $261-268$.

7. Pereirinha, P. G. and Trovão, J. P. (2013) Standardization in Electric Vehicles. http://www.uc.pt/en/efs/research/EESEVS/f/XIICLEEE_1844_PereirinhaTrovao.pdf

8. Wu, H. H., Gilchrist, A., Sealy, K., Israelsen, P., and Muhs, J. (2011) A Review on Inductive Charging of Electric Vehicles. IEEE International Electric Machines and Drives Conference, IEMDC, pp. 866-871. 